\title{
ARQUEOLOGÍA EN LA ESCUELA: EXPERIENCIAS EN EL SECTOR SEPTENTRIONAL DEL NOROESTE ARGENTINO*
}

\author{
ARCHAEOLOGY AT SCHOOL: EXPERIENCES IN THE SEPTENTRIONAL \\ SECTION OF NORTHWESTERN ARGENTINA
}

\author{
Mónica Montenegro ${ }^{1}$
}

\begin{abstract}
En este trabajo presento una experiencia de arqueología pública desarrollada en la provincia de Jujuy, República Argentina. Me interesa compartir representaciones de la comunidad educativa acerca del patrimonio arqueológico local, en un contexto globalizado. Para ello, analizo las configuraciones del pasado y del patrimonio local que se producen en el ámbito escolar, en un marco de emergencia de identidades étnicas. A partir de talleres de expresión artística desarrollados en escuelas primarias, estudio cómo los actores educativos van dando sentido a sus prácticas discursivas. Finalmente, reflexiono acerca de la construcción de conocimientos desde nuevos espacios de de-colonización del saber, considerando a la arqueología pública como marco disciplinar de intervención social en espacios educativos, que promueve la multivocalidad.
\end{abstract}

Palabras claves: arqueología pública, multivocalidad, patrimonio arqueológico, Noroeste Argentino.

In this work I present an experience of developed public archaeology in the province of Jujuy, Argentine Republic. It interests to me to understand the representations of the educative community about the local archaeological heritage, in a globalized context. For it, I analyze the configurations of the past and the local heritage that take place at school, in a conjuncture of emergency of ethnic identities. From artistic workshops developed at primary schools, I study as the educative actors give sense to their discursives' practices. Finally, I reflect about the construction of knowledge from new spaces of de-colonization of the knowledge, considering to public archaeology as a disciplinary frame of social intervention in educative spaces, that promotes multivocality.

Key words: Public archaeology, multivocality, archaeological heritage, Northwest Argentina.

El Noroeste Argentino se ha convertido en las últimas décadas en escenario de activaciones patrimoniales ${ }^{1}$ promovidas desde el Estado nacional. Como respuesta a esa dinámica global, se han producido fenómenos de configuración de identidades y construcción de patrimonio que suelen interpelar la producción de conocimiento científico ${ }^{2}$. Así, han venido sucediéndose una serie de acciones relacionadas con la no exposición de momias y/o restos humanos en museos, demandas por la entrega de cuerpos para reenterrarlos, prohibiciones de excavar lugares que las comunidades consideran sagrados, solicitudes de administración y gestión de sitios arqueológicos, cuestionamiento de la legitimidad del conocimiento científico frente a los saberes populares, y otras. Sin duda, estas acciones afectan el cotidiano devenir de las investigaciones y, en ocasiones, los arqueólogos nos preguntamos ¿qué rol desempeñamos en esta construcción social de la realidad? La misma presenta un sinnúmero de aristas que generan nuevos interrogantes en el campo disciplinar ${ }^{3}$.

El rol de la arqueología en la construcción de discursos acerca del pasado se vuelve una tarea compleja que entraña revisiones, autocríticas y posicionamientos ideológicos. A nivel global, esta problemática ha despertado profundas reflexiones disciplinares, que trascienden el espacio de las excavaciones y el laboratorio. Definitivamente, los nuevos contextos sociopolíticos asociados a la globalización conllevan procesos duales vinculados a homogeneización y fragmentación; el primero se constituye como una dinámica generalizante, en tanto el segundo permite el surgimiento y la visibilización de intereses de grupos minoritarios. La contundencia de estos procesos exigió la revisión de ciertos postulados de

* Artículo seleccionado del conjunto de ponencias presentadas en el Simposio Shifting from Object-centered Research to People-focused Application: Current Approaches to Public Archaeology from Latin America and the Caribbean, realizado en el marco de la 75 reunión anual de la Sociedad de Arqueología Americana, Saint Louis, Missouri, EUA, abril 2010. Este manuscrito fue evaluado por investigadores externos y editado por Reiko Ishihara-Brito y Cameron Griffith, en su calidad de editores invitados de la Revista.

1 Instituto Interdisciplinario Tilcara (FFyL), Universidad de Buenos Aires; Centro Regional de Estudios Arqueológicos (FHyCS), Universidad Nacional de Jujuy. Belgrano 445, Tilcara, Jujuy, Argentina. mmontene@ucn.cl 
la práctica arqueológica ${ }^{4}$ (Hodder 2008; Kim 2008; Layton 1989; Ucko 1989; Trigger 1996).

Por esa época, comenzaron a tomar estado público reclamos de movimientos indígenas en Estados Unidos y Canadá, que demandaban la no intervención de los arqueólogos en sus espacios sagrados y un mayor control sobre su patrimonio. Estas reivindicaciones conllevaron confrontaciones entre científicos e indígenas; como resultado, se generó en estos países un corpus de disposiciones éticas y legales que permitieron una mayor participación de los pueblos originarios en la construcción de narrativas históricas a partir de los objetos (Ayala 2008; Gnecco 2002). Con posterioridad estos procesos fueron observándose en Sudamérica, donde a 500 años de la conquista española se perfilaron nuevos planteos teóricos. Algunos investigadores, alistados en las filas de la arqueología social, apostaron a consolidar una conciencia histórica nacional a partir de sus prácticas ${ }^{5}$, aunque en la generalidad de los países sudamericanos la arqueología siguió conservando un estatus de superioridad en relación con la producción y legitimidad del conocimiento sobre el pasado local, que motivó tensiones entre comunidades indígenas y científicos. No obstante, las relaciones son diferentes y están asociadas a procesos históricos de constitución nacional y a los desarrollos teóricos de la disciplina en los distintos países (Angelo 2005; Ayala 2008; Benavides 2004; Capriles 2003; Funari 1999; Gnecco 2002; Mamani 1989; Menezes Ferreira 2010; Politis 1995).

En Argentina, la relación entre arqueólogos y comunidades ha sufrido profundas rupturas vinculadas fundamentalmente con la resignificación del pasado prehispánico y su relación con el patrimonio arqueológico (Bellelli 2006; Endere et al. 2004; Guráieb y Frère 2008; Laguens 2008; Montenegro 2010; Montenegro y Rivolta 2010; Rocchietti 2004; Rolandi et al. 2004). En un contexto más local, algunos arqueólogos hemos asumido el desafío de generar espacios multivocales de construcción de conocimientos $^{6}$, comprendiendo que el presente reclama nuevas miradas sobre el pasado, y, en esa dinámica, la arqueología pública deviene una vía posible para recrear vínculos con las comunidades locales (Montenegro 2010; Montenegro y Aparicio 2008; Montenegro y Rivolta 2010).

\section{Arqueología Pública: Una Vía de Participación en la Construcción Social del Pasado}

Entiendo a la arqueología pública como una vía posible de reflexión de la práctica arqueológica, pero también de investigación y de participación social. Adhiero particularmente a la definición de Carol McDavid (2002), quien indica que se trata de un fértil campo de investigación arqueológica que incorpora abordajes teórico-prácticos en campos donde los arqueólogos y sus públicos interactúan, como ser: gestión del patrimonio, educación de museos, arqueología y educación. En tal sentido, la considero un campo disciplinar que aspira a promover un acercamiento entre el público y las evidencias materiales del pasado de la región a partir de diversas acciones: pedagógicas, de transferencia, de mediación cultural y de comunicación social, en virtud de los cuales participa directa o indirectamente en los procesos de construcción social del patrimonio arqueológico.

Patrimonio es un concepto que posee una valoración social creciente; esto puede visualizarse tanto en las políticas que propician organismos internacionales como $\mathrm{UNESCO}^{7}$ como en las reapropiaciones y revaloraciones que realizan las comunidades locales ${ }^{8}$. En este escenario, la arqueología adquiere nuevos horizontes de trabajo y entre sus objetivos apuesta a expandir más allá del medio científico los últimos descubrimientos arqueológicos, de manera tal que pueda servir para combatir la depredación de bienes culturales por negligencia o ignorancia (Saucedo Segami 2006). Un campo disciplinar que surge como alternativa es la arqueología pública, y uno de los espacios de intervención, la educación (Merriman 2004; Schadla-Hall 2004; Smardz y Smith 2000). En tal sentido, una experiencia relevante es la que ha venido desarrollando desde el año 2002 el equipo de investigaciones del Proyecto Catalhoyüc ${ }^{9}$, bajo la dirección general de Ian Hodder, quien a través de su escuela de verano ha logrado revitalizar los lazos entre arqueología y comunidad local.

Por lo demás, la inclusión de la arqueología entre los contenidos educativos escolares se ha tornado un tema de interés para los arqueólogos. Sin embargo, llevar la arqueología al aula significa un proceso complejo, que trasciende los espacios de investigación arqueológica (Högberg 2007; Stone y Molineaux 1994). El objetivo es compartir los resultados de las investigaciones científicas a partir del diseño de contenidos curriculares que puedan ser desarrollados en la escuela y aprehendidos por un nuevo público: los niños. Experiencias significativas son los trabajos de Högberg (2007) en escuelas de Suecia y los de Fernández Murillo (2003) para Bolivia. En Argentina, el primer trabajo que analizó la relación entre arqueología y educación fue el de 
Irina Podgorny (1994), proponiendo la inclusión de la arqueología pampeana en la educación argentina. Otra publicación que acercó elementos de arqueología regional a la escuela fue el de María Esther Albeck y colaboradores (1996), realizada específicamente para la Quebrada de Humahuaca; y finalmente podemos citar el trabajo de Rossana Ledesma (2006), para la provincia de Salta, que aborda el tema de arqueología y educación, pero en este caso vinculado al patrimonio y al turismo.

En el caso de la presente investigación, la arqueología pública me brindó un marco conceptual apropiado para analizar críticamente los fenómenos sociales de construcción del patrimonio arqueológico. Se constituyó en una perspectiva teórica desde la cual diseñar propuestas pedagógicas para fortalecer el reconocimiento y la valoración social de la arqueología como la ciencia social que estudia las evidencias culturales del pasado local. Estas acciones permitieron acercar la disciplina al aula, promoviendo un espacio de diálogo intercultural, donde los distintos actores sociales pudieran participar de la construcción del pasado de forma más equitativa.

\section{Arqueología Pública y Educación Intercultural: Nuevas Tramas de Intervención Social}

La provincia de Jujuy está emplazada en el sector septentrional de la República Argentina. Limita al norte con la República de Bolivia; al oeste con las Repúblicas de Chile y Bolivia; al este y al sur con la provincia de Salta. Se trata de una provincia ubicada en área de triple frontera, es por ello un espacio altamente dinámico, donde se desarrollan intensos procesos de configuración de identidades y patrimonio. La interacción cultural promueve relaciones de pertenencia y exclusión a determinados grupos de la sociedad, que están signadas por trazos hegemónicos; las mismas definen una asimetría permanente entre pobladores locales e inmigrantes, donde los elementos culturales propios y ajenos adquieren diferente reconocimiento y valoración social.

En los espacios educativos formales de esta provincia la arqueología es una ciencia poco conocida y/o valorada. En investigaciones anteriores (Montenegro y Aparicio 2008, 2010) hemos constatado que sus objetivos y metodología se encuentran desdibujados en las representaciones sociales de la comunidad educativa. Esto sin duda obedece a la implementación de políticas educativas que invisibilizaron gran parte de su campo de estudio, a través del currículum oficial. Tradicionalmente, en las áreas de frontera de la República Argentina el sistema educativo operó como elemento de homogeneización cultural del Estado nacional; los modelos educativos asimilacionistas contribuyeron en buena medida a acentuar la exclusión cultural. En ocasiones, la condición para permanecer dentro del sistema fue desconocer -al menos en los espacios educativosla identidad cultural y permitir una enculturación a través del currículum oficial. Como resultado, fueron dejándose de lado elementos culturales locales y regionales en aras de la conformación de la "identidad nacional argentina". El patrimonio estuvo constituido fundamentalmente por ideas y prácticas definidas por las aristocracias conservadoras gobernantes ${ }^{10}$. En tanto los discursos acerca del pasado fueron tarea de la historia, que remitió necesariamente al período de conformación del Estado nacional, donde fue invisibilizándose sistemáticamente a las poblaciones originarias, y con ello, a las evidencias materiales del pasado precolombino.

Sin embargo, en el curso de las últimas dos décadas la educación argentina ha experimentado procesos de transformación que proponen mejorar la calidad educativa a partir de la promoción, el desarrollo y la formación integral de los educandos tanto en la faz académica como en la sociocultural. La educación intercultural ha venido imaginándose desde algunos colectivos sociales como un espacio posible para este propósito. De todos modos, transitar la interculturalidad ${ }^{11}$ en el sistema educativo no es tarea sencilla; en el caso particular de Jujuy, significaría considerar una readecuación del currículo en base al contexto regional que permita desarrollar acciones educativas inclusivas, respetando las diversas expresiones culturales presentes en esta provincia. Desde esta óptica, el aporte desde la arqueología pública se enfocó en el diseño de estrategias pedagógicas interculturales, que sirvieran para promover en la escuela espacios multivocales de construcción de discursos sobre el pasado y el patrimonio arqueológico local.

Supuse que estas acciones permitirían rescatar la arqueología del desconocimiento o tal vez del 
olvido, al que tantos años estuvo sometida en el currículum escolar oficial. La premisa fue lograr una participación no sólo simbólica, sino real de la comunidad educativa. Asumí que la arqueología no podía permanecer al margen de los procesos de configuración de patrimonio e identidades locales; era necesario repensar los vínculos entre arqueología y educación. Decidí entonces modificar ciertos argumentos que subyacen las prácticas pedagógicas escolares, instalando una crítica a ciertos contenidos referidos al pasado, para propiciar un debate $\mathrm{y}$, a partir de allí, generar una concientización sobre el tratamiento de la diversidad en el aula. Seleccioné a la escuela primaria como espacio de investigación e intervención, considerando que en la misma se producen fenómenos de apropiación y significación de contenidos, pero también de formación de actitudes.

\section{Relato de una Experiencia Escolar}

La experiencia que comparto a continuación, es el resultado de un conjunto de propuestas pedagógicas para acercar contenidos arqueológicos al aula, desarrolladas durante los ciclos lectivos 2007 y 2008, en la Escuela Primaria No 19: "Delfin Puch", de la localidad de San Antonio (provincia de Jujuy). El propósito fue favorecer una reflexión acerca del pasado y el patrimonio local, y promover actitudes de respeto por la diversidad cultural en el marco del proceso escolar $^{12}$. En función del mismo, el trabajo se basó en un abordaje cualitativo; la metodología de investigación contempló fuentes etnográficas y escritas. Consideré como unidad de estudio a la comunidad educativa primaria y la estrategia de investigación consistió fundamentalmente en acceder a las prácticas discursivas de los actores a través de observación participante, entrevistas, técnicas de expresión grafo-plástica y cuestionarios de opinión. El análisis de documentación escolar oficial me permitió conocer normativas y contenidos relacionados con dos categorías centrales de la investigación: patrimonio y arqueología.

Organicé mi trabajo en dos etapas: en la primera, buscamos acceder a las representaciones de docentes y alumnos acerca de la arqueología; en la segunda, diseñamos junto a los docentes estrategias pedagógicas para acercar contenidos arqueológicos al aula.

\section{Etapas del Trabajo}

\section{Primera etapa: acceso a las representaciones acerca de la arqueología}

Realicé entrevistas a directivos y docentes para saber si anteriormente habían trabajado en la escuela contenidos referidos a arqueología; reproduzco algunos comentarios:

Temas relacionados con el patrimonio se han venido trabajando desde hace dos años, sobre todo se ha estudiado la Quebrada de Humahuaca... Pero de arqueología, no se sabe nada en la escuela... y en la región tampoco tengo conocimiento que se trabajen esos contenidos (Docente 1, comunicación personal 24.10.2007).

Yo no he sabido que se estudien contenidos de arqueología en el grado, porque es muy difícil de explicar a los niños. Son temas que a nosotros nos cuestan un poco porque tampoco tenemos demasiada información (Docente 7, comunicación personal 24.10.2007).

La arqueología me parece un tema importante, sobre todo por el turismo... porque el turismo se acrecienta con la posibilidad de conocer, ver los yacimientos arqueológicos de un lugar... Y sería interesante saber si en San Antonio hay algún lugar importante como los que tiene la quebrada (Docente 3 , comunicación personal 24.10.2007).

Consulté si estaban interesados en trabajar en la escuela temas referidos a la arqueología:

Nosotros estamos interesados en conocer la arqueología de Jujuy, porque, no es un tema muy conocido para nosotros los maestros... Y creo que tampoco se difunden mucho estos estudios... Igual no espere que todos los maestros se interesen porque algunos no tienen tiempo, vienen de Jujuy, y se van enseguida... Podría conversar con los del segundo ciclo, ellos tienen que estudiar los indígenas, así que por ahí se interesan... Igual, en esta 
escuela estamos muy comprometidos con el cuidado del medio ambiente, ya hemos realizado algunos proyectos que han salido muy bien, y se puede trabajar con los niños... el medio ambiente es un eje transversal en esta escuela, y eso también podría incluir la arqueología, ¿no? (Docente 1, comunicación personal 11.10.2007).

Es un tema que me interesa particularmente, la arqueología es apasionante, con todos esos descubrimientos... Pero aquí en Jujuy se conoce muy poco de estas cosas; yo pienso que a los niños les va a gustar trabajar con otros libros o cosas que usted les pueda mostrar de cómo vivían los indios (Docente 5 , comunicación personal 11.10.2007).

Si usted nos trae información a mí me gustaría enseñar a los niños esos temas porque nos interesan a todos... pero a veces no hay tanta información, lo que más se conoce es el Pucara de Tilcara, algunos grados a veces van de viaje de estudios, pero no sabemos mucho más que eso (Docente 7, comunicación personal 17.10.2007).

Una docente comentó que estaba coordinando un proyecto escolar, llamado "Patrimonio de la Humanidad":

El proyecto es grande y por ello estuvimos mucho tiempo pensando cómo lo íbamos a hacer. Lo que queremos es estudiar las características de la Quebrada de Humahuaca desde el punto de vista geográfico y cultural... Entonces pensamos estudiar las formas geológicas, la geografía, su historia y también nos interesaría ver algo del folklore... A fin de año, estamos pensando hacer un viaje, como actividad pedagógica integradora, con los cuartos grados. Vamos a ir a la Quebrada de Humahuaca... para que los niños conozcan nuestro patrimonio, el patrimonio de Jujuy, que ahora es de la Humanidad... Ahí van a conocer las bellezas naturales y las ruinas de los indígenas en Tilcara, que debieron ser muy importantes... (Docente 2, comunicación personal 17.10.2007).
Me interesaba saber si en el contexto local estaban produciéndose fenómenos de construcción del patrimonio que incluyeran materiales o sitios arqueológicos. Pregunté cuál era el patrimonio de San Antonio:

En San Antonio, la cultura gaucha, podemos pensar que es nuestro patrimonio y con orgullo lo digo (Docente 5, comunicación personal 14.11.2007).

Nuestro patrimonio es diferente al de los quebradeños, nosotros somos gauchos, tenemos nuestras fiestas, y nuestras costumbres... la misma música es distinta; aquí por ejemplo se baila folklore, y si no, vea la cantidad de academias de folklore que hay... son como cuatro y todos los niños, desde chiquitos ya saben bailar (Docente 6 , comunicación personal 16.11.2007).
Ah, pero si usted quiere ver qué es el pa- trimonio de San Antonio, entonces tiene que venir para el festival del Quesillo... ahí va ver nuestro folklore, las agrupaciones tradicionalistas, porque San Antonio ha sido siempre de tradición gaucha, acá se ha defendido la patria, en los tiempos de la independencia (Docente 7, comunicación personal 16.11.2007).

Para conocer si estaban produciéndose en esta región fenómenos identitarios de base étnica que pudieran generar apropiación y significación de bienes arqueológicos, pregunté si en San Antonio había comunidades indígenas:

Y, en la quebrada sí; hemos escuchado que ahora hay muchas comunidades indígenas, pero acá no, acá no hay indígenas, somos todos gauchos, los gauchos de San Antonio (Docente 3, comunicación personal 16.11.2007).

Usted sabe que por ahora no hemos visto comunidades originarias en San Antonio, pero igual a mí me parece difícil, porque acá la gente se identifica con la tradición... los gauchos (Docente 4, comunicación personal 16.11.2007). 
Por ahora los indígenas están lejos de nuestra historia, aquí en San Antonio la gente habla de las épocas de la independencia... la tradición está más ligada a los gauchos que defendieron las fronteras de la patria (Docente 5, comunicación personal 22.11.2007).

Pregunté si tenían noticias de la existencia de algún sitio o materiales arqueológicos en los alrededores de San Antonio, y nos comentaron:

\section{Sí, a veces aparecen algunas cosas como morteros de piedra, o esas piedras más achatadas que sirven para moler el maíz, salen cuando pasa el arado... Pero a mí me parece que la gente no sabe qué hacer, porque a veces las veo tiradas en el patio de las casas... (Docente 7, comunicación personal 22.11.2007).}

Aparecen algunas cosas de los indios... morteros y algunas ollitas de barro... no sé, nadie sabe de quién eran, parece que de los indios porque son antiguas (Docente 3 , comunicación personal 22.11.2007).

La verdad que en esta zona, en los valles, no tenemos yacimientos tan lindos como los de la Quebrada, porque ahí sí, estaban los indios omaguacas, y tenían verdaderas fortalezas (Docente 4, comunicación personal 22.11.2007).

\section{Segunda etapa: diseño de propuestas pedagógicas}

Las representaciones de los docentes sirvieron como punto de partida para pensar estrategias pedagógicas. Consideré a la interculturalidad como eje vertebrador del proceso educativo, tomando como referencia las sugerencias de Rubinelli (2010), quien señala que dicho proceso debe constituirse mediante avances conscientes, intencionados y planificados, a partir de la participación en actividades que promuevan el diálogo como base de la interacción y la aceptación de la alteridad para desarrollar la reciprocidad como modalidad de relación. Aposté a generar un espacio multivocal, donde pudieran expresarse los resultados de investigaciones arqueológicas, los contenidos curriculares, los saberes escolares y los conocimientos previos de los niños.

Diseñamos algunas actividades pedagógicas intrainstitucionales y decidimos implementar una de ellas. Se trataba de un taller sobre arqueología local que tuvo dos tópicos de interés: "Importancia de la arqueología para la construcción del pasado local" y "Rol profesional del arqueólogo". Los objetivos fueron: re-conocer las evidencias materiales que dejaron las sociedades del pasado, para re-conocer la diversidad cultural de la provincia de Jujuy; y descubrir diferentes formas de acercarse al conocimiento del pasado local desde la escuela. Los beneficiarios del taller se seleccionaron a partir de muestreo no probabilístico, de tipo muestra de oportunidad. Correspondió al quinto grado $\mathrm{A}$, quinto grado $\mathrm{B}$, sexto grado A y sexto grado $\mathrm{B}$, con un total de 64 niños y sus docentes de: Lengua, Música, Artes Plásticas y Ciencias Sociales. Los docentes consideraban que estas acciones contribuirían a que los niños desarrollaran las siguientes competencias: aprehensión de conceptos relacionados a la arqueología como ciencia, e incorporación de contenidos arqueológicos en los procesos de construcción del pasado local. Mi apuesta pedagógica se centró en favorecer nuevas comprensiones en relación al pasado y al patrimonio arqueológico. Los contenidos que se presentaron en el taller fueron coordinados de modo tal que no resultaran incompatibles con los que establece el Diseño Curricular General de la Provincia de Jujuy (Secretaría de Educación 1997):

- Contenidos conceptuales: Arqueología como ciencia que permite conocer el pasado local. Breve periodización de los desarrollos culturales prehispánicos de la región. Período Inka en la región.

- Contenidos procedimentales: Exposición teórica a cargo de docentes, lectura de material bibliográfico, producción grafo-plástica, presentación y análisis de las producciones en plenario.

- Contenidos actitudinales: Favorecer el encuentro entre el conocimiento arqueológico y la comunidad educativa. Desarrollar actitudes de respeto por la diversidad cultural. Re-conocer las evidencias materiales del pasado prehispánico como información que permite comprender el modo de vida de las sociedades que habitaron el territorio en el pasado. 
Los talleres comprendieron un total de cuatro (4) encuentros durante los cuales trabajamos junto con docentes y estudiantes desarrollando una metodología asimilable a la técnica de grupo focal, para promover una instancia participativa. Los mismos se iniciaban con preguntas orientadas a promover un debate en relación a dos ejes principales: patrimonio local y arqueología. Los niños trabajaron en grupo, y obtuvimos un total de 64 trabajos (dibujos y textos gráficos), que fueron considerados productos comunicativos; en tal sentido, fueron clasificados y analizados utilizando técnicas de investigación en comunicación social para análisis de imágenes. Asumimos una modalidad de exploración transversal extensiva implementando una estrategia de búsqueda de tipo cualitativo consistente en la implementación de estrategias semiestructuradas; específicamente utilizamos guías de observación que permitieron apreciar, seleccionar y registrar elementos grafo-plásticos relacionados con las categorías construidas a priori (arqueología, patrimonio, pasado) y subcategorías que fueron desprendiéndose del análisis de la información (folklore, gauchos, fiestas patronales entre otras); las mismas fueron analizadas y cuantificadas.

\section{Resultados}

En relación al patrimonio local, el 70\% de los alumnos considera que el patrimonio de San Antonio son los gauchos y el 30\% restante que es el folklore (donde se engloban elementos asociados también a los gauchos, como las fiestas patronales, las danzas tradicionales, indumentaria y gastronomía). Las representaciones acerca de la arqueología mostraron que esta ciencia era bastante desconocida en la escuela. Antes de la realización de los talleres, el 96\% de los niños no sabían qué era la arqueología (un 79\% la confundían con paleontología, un 17\% con buscadores de tesoros); solo el $4 \%$ conocía, a través de un documental sobre las tareas de los arqueólogos en relación al estudio de las momias. Los docentes nos hicieron saber que no se consideran dentro del currículum oficial temas relacionados con la arqueología de esta región de la provincia.

La modalidad de taller favoreció la transposición didáctica (Figura 1), en consecuencia, los contenidos

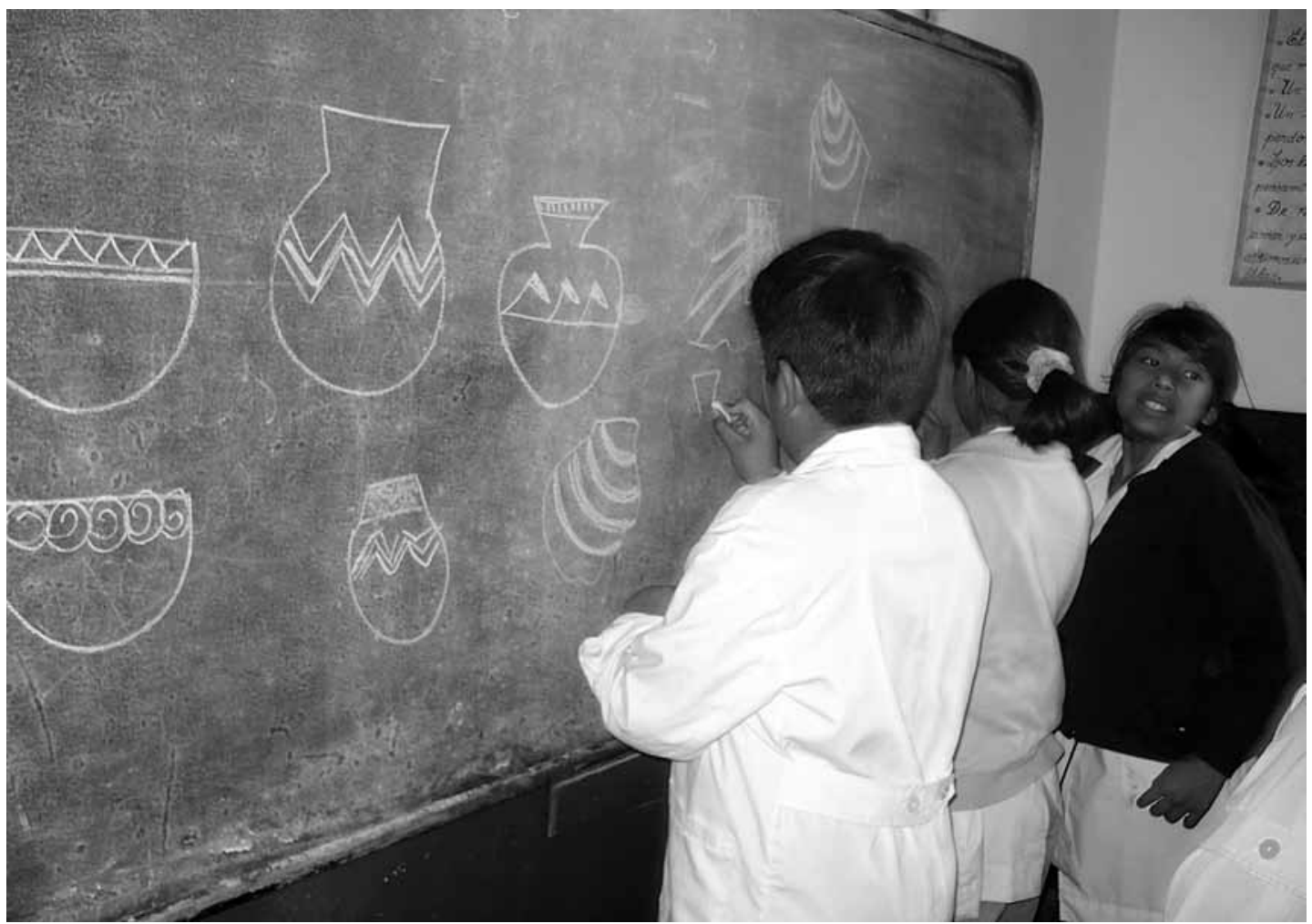

Figura 1. Desarrollo de un taller.

Development of a workshop. 


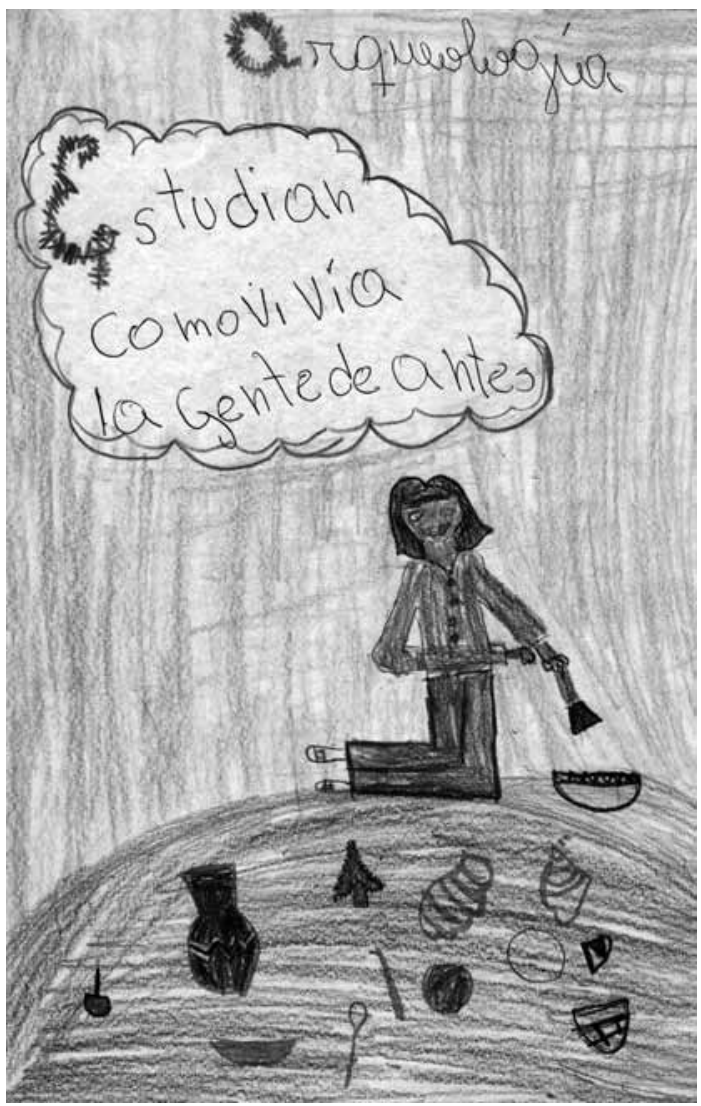

Figura 2. Arqueología: estudian cómo vivía la gente de antes (Érica, Q. San Antonio).

Archaeology: They study how people of the past used to live (Erica, Q. San Antonio).

arqueológicos fueron apropiados en forma exitosa por parte de los alumnos (Figuras 2 y 3 ). Los temas que despertaron mayor interés fueron los referidos a la metodología arqueológica: el 69\% se interesó por conocer cómo se realiza una excavación, el $23 \%$ por el estudio de la cerámica arqueológica y el $8 \%$ por las técnicas de investigación del arte rupestre (Figura 4). Trabajamos aspectos significativos del contexto local, que inciden en la práctica arqueológica, su dinámica y relaciones, a partir de ejes relacionados con la construcción de discursos acerca del pasado local en la escuela.

Se conformó un espacio multivocal, que permitió estudiar el pasado desde tres perspectivas y momentos: los maestros -desde el currículo oficial-hablaron del pasado histórico y la importancia que habían tenido los gauchos de San Antonio durante las guerras de independencia para la conformación del Estado nacional. Los alumnos recordaron relatos orales de sus padres y abuelos que también se relacionaban con la época de independencia. En mi caso, me referí a un pasado más remoto, comentando resultados de las investigaciones arqueológicas en la zona relacionadas principalmente con el período de ocupación incaico en la región (Cremonte et al. 2005; Peralta 2007).

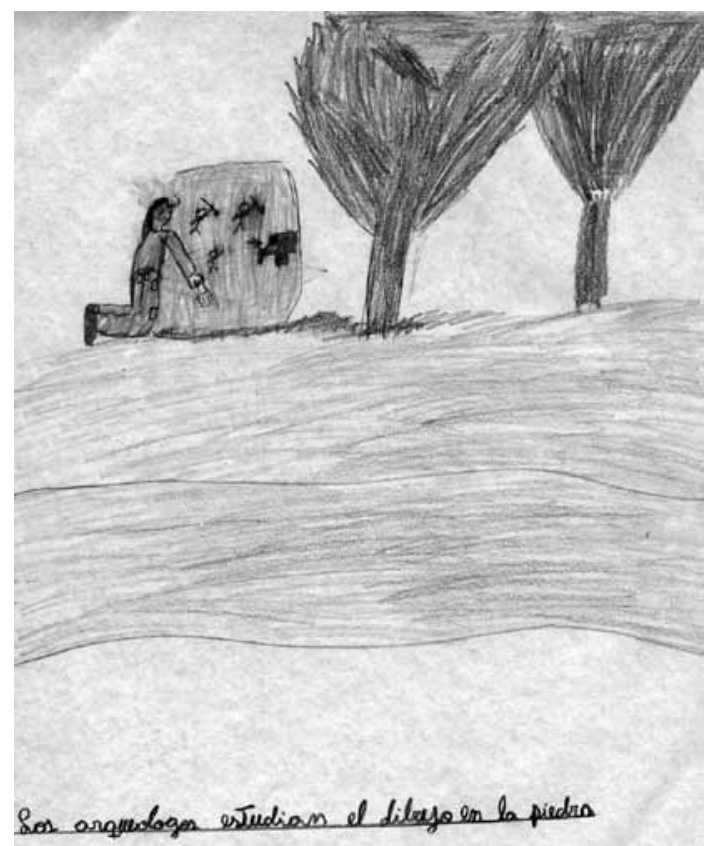

Figura 3. Los arqueólogos estudian el dibujo en la piedra (Nicolás A. San Antonio).

Archaeologists study a drawing on a rock (Nicolás, A. San Antonio).

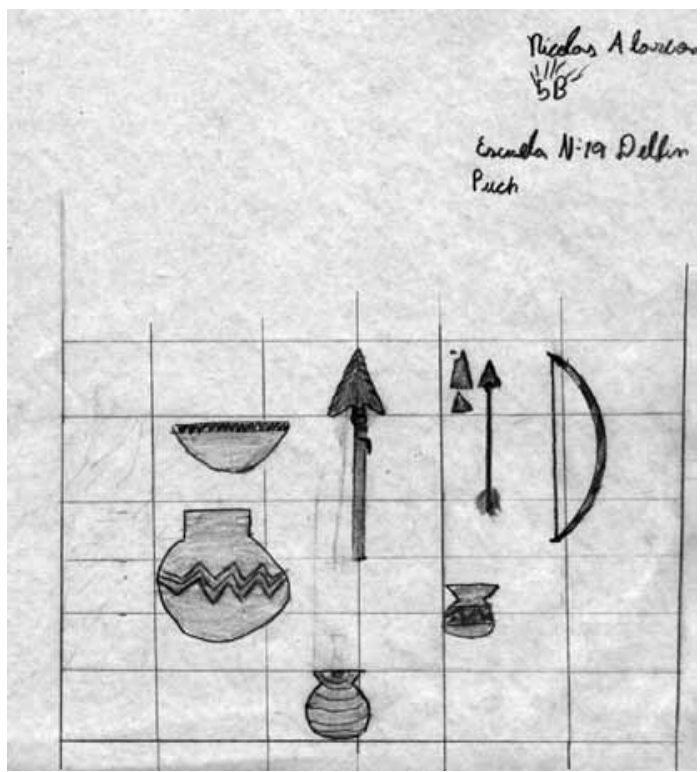

Figura 4. Objetos de los pueblos prehispánicos (Nicolás, A. San Antonio).

Objects of the prehispanic people (Nicolás, A. San Antonio). 
Antes de la realización de los talleres, la historia local estaba absolutamente relacionada con los tiempos de la independencia nacional. Existían leves referencias a épocas tempranas del período colonial, cuando San Antonio era la sede de la aduana en la región. En general, los docentes tenían la convicción de que la mejor forma de acercar a los niños información sobre el pasado prehispánico era visitando la Quebrada de Humahuaca, especialmente el Pucara de Tilcara. Los sitios arqueológicos de San Antonio estaban parcialmente visibilizados, y poseían una escasa valoración para la comunidad educativa local. Sin embargo, al comenzar a compartir los resultados de las investigaciones arqueológicas mostrando las evidencias de ocupación incaica se mostraron muy entusiasmados con conocer las culturas que habitaron esa región en épocas prehispánicas. En tal sentido, los talleres resultaron estrategias pedagógicas para ampliar el horizonte de estudios sobre el pasado local. Se trató de un proceso abierto, que contempló cuestionamientos, diferencias y consensos, que permitió avanzar en el tratamiento de contenidos relacionados con las formas de apropiación y significación del pasado y del patrimonio cultural.

\section{Palabras Finales}

Entiendo que esta propuesta ha servido para acercar contenidos arqueológicos al aula, promoviendo de cierto modo un reconocimiento de la arqueología como ciencia que estudia el pasado. Aunque en un principio esta ciencia resultó desconocida, a medida que fuimos trabajando en los talleres fue despertándose en los niños un gran interés por las actividades que realizan los arqueólogos. Pude advertir que la escuela resulta un espacio social relevante para el desarrollo de procesos de construcción de patrimonio; los mismos están asociados a elementos culturales que sirven para reforzar la identidad local "gaucha". Por lo demás, no registré en esta comunidad educativa apropiación de sitios, ni de bienes arqueológicos, como parte del patrimonio local. Por el momento, las evidencias materiales de ocupaciones prehispánicas son consideradas parte de "otros culturales: los indios", que habitaron antes la zona; un "antes" que se presenta como "pasado" difuso, remoto, con dificultades para acotarlo temporalmente.

Asimismo, las actividades de mediación científica que hemos venido desarrollando evidencian nuevas perspectivas para los proyectos de arqueología pública ligados a educación. A partir de la nueva Ley de
Educación Nacional, la escuela primaria se abre a la comunidad desde un nuevo espacio pedagógico, donde los niños son protagonistas no solo en los procesos de enseñanza y aprendizaje, sino que participan como activos elementos de sensibilización social acerca de problemáticas locales actuales. Pensamos que, desde un enfoque lúdico y pedagógico, es posible acercar contenidos de arqueología que amplíen el horizonte de estudios del pasado en la escuela y que, a su vez, ofrezcan a cada actor educativo, una nueva experiencia de participación logrando un compromiso con la protección del patrimonio arqueológico, en el marco de las relaciones interculturales existentes en la región. De todos modos, será necesario que los contenidos se incorporen oficialmente al Diseño Curricular de Educación General Básica de la Provincia de Jujuy, para que pueda garantizarse un tratamiento "oficial y efectivo", ya que de otro modo sólo serán esfuerzos aislados y efímeros.

Los efectos de las activaciones patrimoniales comienzan a hacerse presente en los escenarios educativos de la provincia de Jujuy. En este contexto, es necesario apostar al desarrollo de acciones educativas que promuevan el reconocimiento del patrimonio arqueológico local en las escuelas y la construcción de un pasado más inclusivo. Las mismas contribuirán además a crear una conciencia de respeto por el patrimonio de otras culturas, fomentando un espacio de interculturalidad. Esto podría redundar en un mejoramiento de la calidad educativa, ya que los aprendizajes estarán relacionados con el contexto sociocultural del que participan los niños, en consecuencia, se volverán significativos.

Agradecimientos: En primer lugar deseo agradecer a Reiko y Cameron, por invitarme a participar de esta publicación. Los trabajos fueron realizados en el marco del PICT2007-Nº 01538 (SECTER, Universidad Nacional de Jujuy), mi agradecimiento a su Directora, María Beatriz Cremonte. Un agradecimiento especial a Calogero Santoro por su apoyo permanente; a María Clara Rivolta por compartir mis reflexiones disciplinares, y a María Elisa Aparicio por su mirada antropológica educativa. Finalmente, deseo agradecer a la Comunidad Educativa de la escuela No 19 "Delfín Puch" de San Antonio, a los docentes: Ana María Díaz, Magda Sivila, María Guadalupe Grágeda, María Mercedes Tévez, Oscar Valencia, y a la Supervisora Lilian Aparicio, por acompañarme en la construcción de un pasado multivocal y más participativo. 


\section{Referencias Citadas}

Albeck, M.E., M. Calvo, A.M. González, H. Portal y L. Viste 1996. Quebrada de Humahuaca: Más de 10.000 Años de Historia. Escuela Normal "Dr. Eduardo Casanova" y Universidad Nacional de Jujuy, Tilcara.

Ángelo, D. 2005. La arqueología en Bolivia. Reflexiones sobre la disciplina a inicios del siglo XXI. Arqueología Suramericana 1(2):185-211.

Apaza, N. 2010. Dictamen sobre el proyecto de ley marco de patrimonio natural y cultural de la Provincia de Jujuy. Equipo Federal del trabajo, Edición No 56. http://www.newsmatic.e-pol. com.ar/index.php?pub_id=99\&sid=4873\&aid $=50978 \&$ eid $=56 \&$ NombreSeccion=Pueblos\%20Originarios \&Accion=VerArticulo (31 agosto 2011).

Ayala, P. 2008. Políticas del Pasado: Indígenas, Arqueólogos y Estado en Atacama. Línea Editorial Instituto de Investigaciones Arqueológicas y Museo, Universidad Católica del Norte, San Pedro de Atacama.

Bellelli, C. 2006. Arqueología y patrimonio. Una historia de usos y abusos en el valle medio del Río Chubut (Patagonia Argentina). En Tramas en la Piedra, editado por D. Fiore y M.M. Podestá, pp. 251-262. Instituto Nacional de Antropología y Pensamiento Latinoamericano y World Archaeological Congress, Buenos Aires.

Benavides, O.H. 2004. Los ritos de la autenticidad: indígenas, pasado y el estado ecuatoriano. Arqueología Suramericana 1(1):5-48.

Capriles, J.M. 2003. Arqueología e Identidad étnica: el caso de Bolivia. Chungara Revista de Antropología Chilena 35:347-353.

Coronil, F. 2000. Naturaleza del poscolonialismo: del eurocentrismo al globocentrismo. En La Colonialidad del Saber: Eurocentrismo y Ciencias Sociales. Perspectivas Latinoamericanas, compilado por E. Lander, pp. 87-112. CLACSO-UNESCO, Buenos Aires.

Cremonte, M.B., M.A. Zaburlin y S. Peralta 2005. Ordenamiento espacial y arquitectura del sitio incaico Agua Hedionda. Cuadernos de la Facultad de Humanidades y Ciencias Sociales 29:23-42. Universidad Nacional de Jujuy, Jujuy.

Endere, M., M.G. Chaparro y V. Williams 2004. Construyendo el pasado en comunidad. El caso de las ruinas de Tolombón. Actas del II Congreso Internacional de Patrimonio Cultural. Córdoba. Versión electrónica.

Fernández Murillo, M.S. 2003. Comunidades locales y la enseñanza de la arqueología: una experiencia en Bolivia. Chungara Revista de Antropología Chilena 35:355-359.

Funari, P. 1999. Brazilian archaeology: A reappraisal. En Archaeology in Latin America, editado por G. Politis y B. Alberti, pp. 17-37. Routledge, Londres.

Gnecco, C. 2002. La indigenización de las arqueologías nacionales. Convergencia 9(27):133-149. Universidad Autónoma del Estado de México, Toluca.

Guráieb, A.G. y M.M. Frère 2008. Caminos y Encrucijadas en la Gestión del Patrimonio Arqueológico Argentino. Editorial de la Facultad de Filosofía y Letras, Universidad de Buenos Aires.

Hodder, I. 2008. Multivocality and social archaeology. En Evaluating Multiple Narratives: Beyond Nationalist, Colonialist,
Imperialist Archaeologies, editado por J. Habu, C. Fawcett y J. Matsunaga, pp. 196-200. Springer, Nueva York.

Högberg, A. 2007. The past is the present - prehistory and preservation from a children's point of view. Public Archaeology 6(1):28-46.

Kim, M. 2008. Multivocality, multifaceted voices, and Korean archaeology. En Evaluating Multiple Narratives: Beyond Nationalist, Colonialist, Imperialist Archaeologies, editado por J. Habu, C. Fawcett y J. Matsunaga, pp. 118-137. Springer, Nueva York.

Kojan, D. 2008. Paths of power and politics: historical narratives at the Bolivian site of Tiwanaku. En Evaluating Multiple Narratives: Beyond Nationalist, Colonialist, Imperialist Archaeologies, editado por J. Habu, C. Fawcett and J. Matsunaga, pp. 69-85. Springer, NuevaYork.

Laguens, A. 2008. Tiempos, espacios y gente: reflexiones sobre las prácticas de la arqueología de Córdoba desde Córdoba, Argentina. Arqueo Web 10. http://www.ucm.es/info/arqueoweb/ pdf/10/laguens.pdf (13 febrero 2012).

Layton, R. 1989. Introduction: conflict in the archaeology of living traditions. En Conflict in the Archaeology of the Living Traditions, editado por R. Layton, pp. 1-21. Routledge, London y Nueva York.

Ledesma, R. 2006. El Patrimonio Antropológico en la Educación Formal, en el Departamento de Cafayate, Salta, Argentina (1996-2003). Tesis de Maestría en Conservación del Patrimonio. Universidad Internacional de Andalucía, Sede La Rábida.

Lumbreras, L. 1990. La arqueología sudamericana, tres décadas. Revista Española de Antropología Americana 20:57-66.

Mamani, C. 1989. History and prehistory in Bolivia: what about the Indians? En Conflict in the Archaeology of the Living Traditions, editado por R. Layton, pp. 46-59. Routledge, London y New York.

McDavid, C. 2002. From Real Space to Cyberspace: The Internet and Public Archaeological Practice. Tesis doctoral, Cambridge University, Cambridge.

Menezes Ferreira, L. 2010. Territorio Primitivo. A Institucionalização da Arqueología no Brasil (1870-1917). EdiPUCRS, Pontificia Universidad Católica do Río Grande do Sul, Porto Alegre.

Merriman, N. 2004. Involving the public in museum archaeology. En Public Archaeology, editado por N. Merriman, pp. 85-108. Routledge, London y New York.

Montenegro, M. 2010. El patrimonio arqueológico de Jujuy: miradas diversas desde la escuela. Estudios Sociales del NOA, nиеva serie 10:107-121. Instituto Interdisciplinario Tilcara, Tilcara.

Montenegro, M. y M.E. Aparicio 2010. Festividades, celebraciones y patrimonio en Jujuy: experiencias desde la escuela. En ¿Los Otros Como Nosotros? Interculturalidad y Ciudadanía en la Escuela. Reflexiones desde América Latina, compilado por M.L. Rubinelli, Tomo II, pp. 61-72. Unidad de Pensamiento Latinoamericano, Relaciones Interétnicas e Interculturales, Universidad Nacional de Jujuy, Jujuy. 
- - - 2008. Los niños y el patrimonio, ¿Una deuda de la Educación? Cuadernos de la Facultad de Humanidades y Ciencias Sociales de Jujuy 34:103-119. Universidad Nacional de Jujuy, Jujuy.

Montenegro, M. y M.C. Rivolta 2010. Re-construyendo el presente desde el pasado: los arqueólogos y sus desafíos. Ponencia presentada en VReunión Internacional de Teoría Arqueológica en América del Sur, Universidad Central de Venezuela, Caracas.

Peralta, S. 2007. Resultados de los análisis arquitectónicos y funcionales del sector alto de Agua Hedionda (Dpto. San Antonio, Jujuy). Actas del XVI Congreso Nacional de Arqueología Argentina, pp. 191-197, Editorial Universidad Nacional de Jujuy, Jujuy.

Podgorny, I. 1994. Arqueología y Educación: La Inclusión de la Arqueología Pampeana en la Educación Argentina. Tesis presentada para optar al título de Doctora en Ciencias Naturales, Universidad Nacional de La Plata, Buenos Aires.

Politis, G. 1985. The socio-politics of the development of archaeology in Hispanic South America. En Theory in Archaeology: A World Perspective, editado por P. Ucko, pp. 197-235. Routledge, Londres.

Prats, LL. 1997. Antropología y Patrimonio. Editorial Ariel, Barcelona.

Rocchietti, A.M. 2004. Crítica de la razón patrimonialista. Actas del XV Congreso Nacional de Arqueología Argentina. Río Cuarto, Córdoba.

Rockwell, E. 1995. La Escuela Cotidiana. Fondo de Cultura Económica, México, D.F.

Rolandi, D.S., C.Aschero, M.M. Podestá y A. Re 2004. Inca Cueva 1: Un siglo de aciertos y desaciertos en un sitio de alto valor patrimonial. En Problemáticas de la Arqueología Contemporánea, Tomo 1, compilado por A. Austral y M. Tamagnini, pp. 99-108. Universidad Nacional de Río Cuarto, Río Cuarto.

Rubinelli, M.L. 2010. Algunas cuestiones acerca de una educación intercultural. En ¿Los Otros Como Nosotros? Interculturalidad y Ciudadanía en la Escuela. Reflexiones desde América Latina, Tomo II, compilado por M.L. Rubinelli, pp. 37-60. Unidad de Pensamiento Latinoamericano, Relaciones Interétnicas e Interculturales, Universidad Nacional de Jujuy, Jujuy.

Saucedo Segami, D. 2006. Arqueología pública y su aplicación en el Perú. Revista Electrónica de Arqueología 1(1). http://mileto. pucp.edu.pe/arkeos//images/documentos/articulos/1-art-ds.pdf (14 febrero 2012). Pontificia Universidad Católica del Perú, Lima.

Schadla-Hall, T. 2004. The comforts of unreason: the importance and relevance of alternative archaeology. En Public Archaeology, editado por N. Merriman, pp. 255-271. Routledge, Londres y Nueva York.

Secretaría de Educación 1997. Diseño Curricular de Educación General Básica. Segundo Ciclo. Secretaría de Educación, Ministerio de Gobierno, Justicia y Educación de la Provincia de Jujuy, San Salvador de Jujuy.

Smardz, K. y S.J. Smith (eds.) 2000. The Archaeology Education Handbook: Sharing the Past with Kids. Altamira Press, Walnut Creek, California.

Stone, P. y B. Molineaux (eds.) 1994. The Presented Past. Heritage, Museums and Education. One World Archaeology 25. Routledge, Londres.

Trigger, B. 1996. Alternative archaeologies: nationalist, colonialist, imperialist. En Contemporary Archaeology in Theory, editado por R. Preucel y I. Hodder, pp. 615-631. Blackwell Publishers, Oxford.

Ucko, P. 1989. Foreword. En Conflict in the Archaeology of the Living Traditions, editado por R. Layton, pp. VIII-XVI. Routledge, Londres y Nueva York.

Walsh, C. 2009. Interculturalidad, crítica y pedagogía intercultural: in-surgir, re-existir y re-vivir. En Educación Intercultural en América Latina. Memorias, Horizontes Históricos y Disyuntivas Políticas, coordinado por P. Medina Melgarejo, pp. 25-42. Editorial Plaza y Valdés, México, D.F.

\section{Notas}

1 Prats (1997) sostiene que el patrimonio como construcción social incluye un repertorio de referentes patrimoniales activado por versiones ideológicas de una determinada identidad; en tal sentido, y dado que pueden coexistir distintas versiones de una misma identidad articuladas a través de relaciones de complementariedad u oposición, en ocasiones el patrimonio se constituye en un campo de confrontación simbólica inevitable entre las distintas versiones concurrentes. La correlación entre intereses, valores y situaciones históricas cambiantes permite entender a las activaciones patrimoniales como estrategias, donde el principal agente de activación es el poder político.

2 Así, en espacios sociales localizados bajo condiciones globales, las identidades colectivas se están construyendo en formas inéditas a través de una articulación compleja de fuentes de identificación tales como la religión, territorialidad, raza, clase, etnicidad, género y nacionalidad, pero ahora esta articulación está informada por discursos universales de derechos humanos, leyes internacionales, ecología, feminismo, derechos culturales y otros medios de hacer respetar las diferencias dentro de la igualdad (Coronil 2000).

3 Sin embargo, esta situación parecería no ser exclusiva de la arqueología; como sugiere Coronil (2000) el proceso conocido como globalización incluye nuevos campos de lucha teórica y práctica, y está desestabilizando no sólo las fronteras geográficas y políticas, sino también los protocolos disciplinarios y sus paradigmas teoréticos.

4 Trigger (1996) advirtió que los pueblos originarios de países en desarrollo consideraban cada vez más inadecuada y neocolonialista la idea de que los datos arqueológicos debieran utilizarse sólo para formular y probar un conjunto de teorías generales sobre el comportamiento humano y el cambio cultural.

5 "Los que hacemos arqueología sudamericana somos claramente conscientes de que nuestra tarea compromete 
directamente nuestros intereses históricos inmediatos[...] Por eso, la arqueología es para nosotros, además de reconstrucción histórica y tarea científica, una búsqueda de afirmación de identidad que nuestros pueblos tienen confundida detrás de un síndrome provocado por la razón colonial" (Lumbreras 1990:64).

6 Espacios donde se desarrollan abordajes vinculados a la multivocalidad, entendida en términos amplios como la idea de que existen siempre múltiples interpretaciones del pasado que permiten que sectores, otrora marginados, puedan efectuar interpretaciones utilizando sus propios términos y aproximaciones a fin de construir significados diferentes a los modelos dominantes (Hodder 2008; Kojan 2008).

7 Definidos específicamente por la Conferencia General de la Organización de las Naciones Unidas para la Educación, la Ciencia y la Cultura, en su 17a reunión celebrada en París del 17 de octubre al 21 de noviembre de 1972. http://whc. unesco.org/archive/convention-es.pdf.

8 Un ejemplo notorio lo constituye el Dictamen sobre el proyecto de ley marco de patrimonio natural y cultural de la provincia de Jujuy, que realiza la jurista Nimia Apaza (2010).
9 El programa "Escuela de verano Catalhoyüc", coordinado por Shahina Farid, promueve la educación en arqueología en las escuelas. Su propósito es contribuir a la educación de los jóvenes de la región acerca de la importancia de la arqueología, intentando que la comunidad local conozca mejor su patrimonio y se haga responsable de su cuidado.

10 Entre los cuales se encontraban los símbolos patrios (bandera, escudo, himno y escarapela).

11 Interculturalidad puede entenderse como un proceso de relación, comunicación y aprendizaje entre personas, grupos, conocimientos, valores y tradiciones distintas, enfocado en general a construir y propiciar un respeto mutuo y un desarrollo pleno de las capacidades individuales a pesar de sus diferencias (Walsh 2009).

12 Entendido como un conjunto de relaciones y prácticas institucionalizadas históricamente, dentro del cual el currículum oficial es un nivel normativo. Está conformado por la interacción de tradiciones históricas, variaciones regionales, decisiones político-administrativas y burocráticas, consecuencias imprevistas en la planificación técnica e interpretaciones particulares que hacen maestros y alumnos de los elementos en torno a los cuales se organiza la enseñanza (Rockwell 1995). 